\title{
Predictors of disengagement from Early Intervention in Psychosis services
}

Francesca Solmi, Abdolali Mohammadi, Jesus A. Perez, Yasir Hameed, Peter B. Jones and James B. Kirkbride

\section{Background}

The effectiveness of Early Intervention in Psychosis (EIP) services for individuals with a first episode of psychosis (FEP) could be thwarted by high rates of early disengagement.

\section{Aims \\ To investigate which factors predict disengagement with EIP services.}

\section{Method}

Using data from a naturalistic cohort of 786 EIP clients in East Anglia (UK), we investigated the association between sociodemographic and clinical predictors and disengagement using univariable and multivariable cox proportional hazards models.

\section{Results}

Over half (54.3\%) of our sample were discharged before receiving 3 years of EIP care, with 92 (11.7\%) participants discharged due to disengagement. Milder negative symptoms, more severe hallucinations, not receiving an FEP diagnosis, polysubstance use and being employed were associated with greater disengagement.

\section{Conclusions}

Our findings highlight heterogeneous reasons for disengagement with EIP services. For some patients, early disengagement may hinder efforts to sustain positive long-term EIP outcomes. Efforts to identify true FEP cases and target patients with substance use problems and more severe positive symptoms may increase engagement.

\section{Declaration of interest}

None.

\section{Keywords}

Early intervention services; psychosis; cohort study; Social Epidemiology of Psychosis in East Anglia; SEPEA.

\section{Copyright and usage}

(C) The Royal College of Psychiatrists 2018. This is an Open Access article, distributed under the terms of the Creative commons Attribution licence (http://creativecommons.org/licenses/by/4.0/), which permits unrestricted re-use, distribution, and reproduction in any medium, provided the original work is properly cited.
Early Intervention in Psychosis (EIP) services offer phase-specific pharmacological, psychological, social, occupational and educational support to individuals with early or prodromal symptoms of psychosis for up to 3 years. ${ }^{1}$ In England, EIP services typically consist of a multidisciplinary team of experts, including psychiatrists, care coordinators, clinical psychologists, psychological therapists and employment and educational specialists. ${ }^{2}$ They were introduced following demonstration that longer duration of untreated psychosis was associated with adverse clinical, functional and social outcomes. ${ }^{3}$ Over the past 15 years, they have gained increased traction worldwide. ${ }^{4}$ In the UK, studies suggest that EIP services can be effective in halting the transition to psychosis ${ }^{5}$ and are cost-effective., Nevertheless, concerns exist that high rates of early disengagement from EIP services - estimated to range from 13 to $31 \%^{8-11}$ - could hamper their effectiveness, particularly with respect to sustaining long-term positive outcomes. Although studies evaluating the optimal length of EIP service provision have yielded conflicting results, ${ }^{12-15}$ there is some evidence that a longer time spent in EIP is associated with better long-term outcomes. ${ }^{14}$ It is therefore important to identify potential factors - positive or negative - that predict discharge from EIP services before receipt of a full package of care. Two recent literature reviews found that male gender, unemployment, substance use, not having a family member involved in treatment and belonging to an ethnic minority are the most consistently reported predictors of patient disengagement from EIP services. ${ }^{16,17}$ However, the evidence is mixed for other factors such as age, symptom severity and social functioning, as they appear to be associated differently with disengagement from pharmacological therapy versus psychosocial services. For example, studies have shown that lower social functioning and symptom severity as well as older age are associated with disengagement from psychosocial services, whereas the reverse is true for pharmacological treatments. ${ }^{16,17}$ In this study, we sought to investigate sociodemographic and clinical factors that predicted early discharge due to disengagement from six EIP services in the East Anglia region of the UK, using a large, longitudinal data set from the Social Epidemiology of Psychosis in East Anglia (SEPEA) study. Based on findings from the previous literature, we hypothesised that participants with greater substance use, fewer symptoms, and who were male, older and from an ethnic minority background would be more likely to disengage from EIP services.

\section{Method}

\section{Sample}

Individuals referred to six EIP services in East Anglia (UK) for a suspected first episode of psychosis (FEP) between 1 July 2009 and 28 March 2013 were eligible to be included in the SEPEA study if they were: 16-35 years old (or 17-35 years in two EIP services: Cambridgeshire North and South), resident in the catchment area, did not have an intellectual disability or an organic basis to the disorder, and had not been previously in contact with health services for FEP. All participants had suspected psychosis at the time of referral. Details on the characteristics of the catchment area and its representativeness have been provided elsewhere. ${ }^{18}$

All participants were followed up from date of acceptance into EIP services until completion of a 3 year programme of EIP care or discharge from the service, if earlier. In CAMEO North and South the care offering was changed to 2 years for all referrals after 1 October 2013 because of budget constraints. We included all participants accepted onto EIP caseloads, irrespective of later clinical diagnoses (assessed at 6 months following acceptance and at 3 years or discharge, if earlier). In this study, we included all participants who had complete data on the variables of interest. The Cambridgeshire III Local Research Ethics Committee provided ethical approval for this study (09/H0309/39). 


\section{Outcome}

Our primary outcome was time to discharge because of disengagement with services. Participants were considered as having disengaged with services after all possible ways to engage them had been explored by the clinical team. These included: appointment letters, phone calls, text messages, emails, home visits and contact with family, friends and other health, education and social care providers. This process involved several attempts (usually at least six to eight attempts over a 2-3 month period). Once all options to reengage a participant had been exhausted, a decision was made at a clinical team meeting to discharge the person to their general practitioner (GP). The person was then informed of this decision by letter, with a final offer to contact the service if they wished to reengage. Their GP was also informed of this decision.

Participants who were discharged for other reasons - e.g. because of recovery and transfer to primary care, transfer to a different EIP or mental health service, having moved out of the area or for other reasons (including death, transfer to criminal justice, discharge requested by the participant or drug-induced psychosis) were censored at their discharge date, but were not considered as having the outcome of interest. All other participants (40.6\%) were followed until receipt of 3 years of standard EIP care, including a handful of participants $(n=43,5.4 \%)$ who received EIP care for longer than 3 years (median time in care of 39.7 months, interquartile range (IQR) of 37.9-41.4).

\section{Predictors}

We included several sociodemographic and clinical factors previously associated with disengagement from EIP services as potential predictors of disengagement. Sociodemographic data were recorded during the first clinical contact with participants, using a standardised form. We included data on age at referral (16-24, 25-29 and 30-35), gender, ethnicity (White or Black and minority ethnic group, the latter including Black African, Black Caribbean, other Black and minority ethnic groups, Bangladeshi or Pakistani), country of birth (UK or outside the UK), marital status (married or divorced/separated/single) and socioeconomic status (professional, managerial or intermediate; routine or manual; student; unclassified or unemployed).

Participants received an ICD-10 (1992) diagnosis derived by using a two-stage diagnostic procedure comparing clinical and research diagnoses. Clinicians provided a diagnosis at 6 months after EIP acceptance and at discharge. Subsequently, a trained panel of 25 clinicians derived research-based diagnoses from clinical records using 90 standardised symptom items of the Operational Criteria Checklist for Psychotic Illness and Affective Illness (OPCRIT) ${ }^{19}$ a diagnostic instrument known to provide reliable ICD-10 diagnoses $(k=0.70){ }^{20}$ We defined and grouped OPCRITconfirmed diagnoses as follows: schizophrenia and other nonaffective psychoses (ICD-10 codes F20-F29), substance-induced psychoses (ICD-10 codes F10-F19), bipolar disorder and psychotic depression (ICD10 codes F30-F33) or, if there was no indication of FEP, any other non-psychotic psychiatric diagnosis.

Using auxiliary items from OPCRIT, we included data on presence of a family history of schizophrenia or any other mental health disorders, lack of insight (yes/no), substance abuse and dependency (none, one, two or more substances), experience of an acute psychosocial stressor prior to admission and duration of untreated illness (DUI) ( $\leq 1$ month, 2-3 months, 4-6 months, 7-12 months or $>1$ year). DUI was defined as the time elapsed from the emergence of prodromal symptoms (including any active phases of the illness during which non-drug interventions such as cognitivebehavioural therapy may have been received, if those therapies were given because of their potentially antipsychotic properties) to the receipt of the first antipsychotic medication. If the participant did not receive medication, the DUI end-point was defined as time of first non-pharmacological treatment, discharge or time of the 6-month OPCRIT assessment, whichever came sooner. Finally, we included seven standardised indicators of symptom severity (mania, depression, delusions, hallucinations, paranoia, psychomotor poverty and first-rank delusion) following a factor analysis of OPCRIT items to identify psychopathology dimensions in this sample, derived from OPCRIT data. The methodology employed to derive these symptom scores has been described elsewhere (Kirkbride J.B., personal communication 2018).

Finally, we employed two variables to indicate waiting time prior to EIP acceptance, consistent with mandated Access and Waiting Time standards in England ( $\leq 2$ weeks or $>2$ weeks ${ }^{2}$ ) and EIP service (CAMEO North, CAMEO South, West Norfolk EIP, Central Norfolk EIP, Great Yarmouth and Waveney EIP and the former Suffolk EIP).

\section{Data analyses}

Participants contributed to follow-up time from date of EIP acceptance until the end of the 3 -year care package (2 years in CAMEO services for patients discharged after 1 October 2013) or discharge, if sooner. We used Chi-square tests and analysis of variance to describe the study sample and length of time in treatment by reason for discharge ('treatment completed', 'left EIP for other reasons' or 'disengaged with EIP').

Next, we investigated sociodemographic and clinical predictors of disengagement using Cox proportional hazard models. Participants who were discharged early from EIP for reasons other than disengagement $(n=375,47.7 \%)$ were right-censored and so contributed follow-up time to the analyses where they were potentially 'at-risk' of early disengagement. We ran univariable regression models for each potential predictor included in the study, after checking that the proportionality-of-hazards assumption was met. For each univariable model, we estimated Wald-test $P$-values and Akaike's information criteria (AIC) goodness-of-fit parameters. Age, gender, marital status and EIP centre were retained as a priori confounders. All other variables were retained for inclusion in multivariable models if they had $P$-values $\leq 0.05$. We employed a forward-fitting modelling approach, progressively including variables with larger AIC values to the multivariable model and testing superiority of each model, compared with the former, via likelihood ratio test (LRT). In post hoc analyses, we tested whether the effect of any psychotic symptoms which predicted risk of disengagement differed by the presence or absence of comorbid substance misuse, via LRT for statistical interaction, as before. As a sensitivity analysis, we ran the same models but excluded participants who did not complete the EIP care package for reasons other than disengagement from the analysis. We also re-ran the analyses using a logistic regression model (outcome: $0=$ completed treatment package/left for any reason other than disengagement, $1=$ disengaged) as a sensitivity analysis, since this did not include length of time in treatment as an assumption. All analyses were performed using Stata version $13 .^{21}$

\section{Results}

\section{Sample}

We included 786 participants in our sample, representing $98.5 \%$ of the whole SEPEA study sample $(N=798)$ accepted into EIP care. A total of $12(1.5 \%)$ people were excluded because of missing data on country of origin $(n=1)$, discharge date $(n=1)$, discharge reason $(n=2)$ or marital status $(n=8)$. The overall median time under EIP care was 25.5 months $(\mathrm{IQR}=13.3-35.9)$. 
As shown in Table 1, the majority of the sample was male, younger than 25 years, of White British ethnicity, born in the UK, single/divorced/separated, unemployed, had a diagnosis of schizophrenia or other non-affective psychosis, used one or more drugs, had waited longer than 2 weeks to enter treatment and had over 6 months of untreated illness.

\section{Rate and predictors of disengagement}

A total of 467 (59.4\%) participants were discharged early from EIP services in our region (Table 2). Of these participants, 40 (5.1\%) were transferred to EIP services outside the region (meaning that at least $54.3 \%$ of our sample were discharged before receiving a full EIP care package) and $92(11.7 \%)$ were discharged due to disengagement, our primary outcome. Other reasons for discharge ae shown in Table 2 and in a flow chart presented in Supplementary Figure 1 available at https://doi.org/10.1192/bjp. 2018.91. Participants who were discharged because they had moved out of the area had the shortest follow-up time (median 5.6 months, IQR 3.8-10.4). Participants who left the programme due to disengagement had a median follow-up time of 15.0 months (IQR 8.2-21.2) (Table 2).

Univariable analyses (Table 1) showed that participants who were not diagnosed with FEP were more likely to be discharged due to disengagement compared with those diagnosed with non-affective psychosis. Participants in employment, with one or polysubstance misuse/dependency and those who had greater hallucinatory symptoms were also at greater risk of being discharged early from EIP services due to disengagement. Univariable analyses also suggested that participants with more severe manic symptoms, severe psychomotor poverty and first-rank delusions and a diagnosis of affective psychosis were at reduced risk of disengagement. Disengagement did not vary by other characteristics, including ethnicity, EIP service and family history of mental illness (Table 1).

Following multivariable modelling (Table 3), we found evidence that participants who did not receive an FEP diagnosis (hazard ratio 2.52, 95\% CI 1.49-4.26), who had a DUI of between 5 and 8 weeks (hazard ratio 5.19, 95\% CI 1.85-14.56) and who had a history of polysubstance misuse ( $\geq 2$ drugs; hazard ratio 2.20 , 95\% CI $1.30-$ 3.72) were at greater risk of disengagement from EIP services. Those who were unemployed (hazard ratio 0.44 , 95\% CI $0.28-$ 0.69 ), with more severe psychomotor poverty (hazard ratio 0.71 , 95\% CI 0.57-0.88) and first-rank delusions (hazard ratio 0.74, $95 \%$ CI $0.60-0.83$ ) were at lower risk of disengagement. We also found weak evidence that men (hazard ratio $1.61,95 \%$ CI $0.98-$ 2.63, $P=0.06$ ), participants with more hallucinations (hazard ratio $1.24,95 \%$ CI $0.97-1.58, P=0.08$ ), those aged $30-35$ years (hazard ratio $1.88,95 \%$ CI $0.95-3.70, P=0.07$ ) and those with single substance misuse (hazard ratio $1.66,95 \%$ CI $0.94-2.94, P=$ 0.08 ) were at increased risk of disengagement. In post hoc analyses, we considered whether the increased risk of disengagement associated with more hallucinations differed between those with and without comorbid substance misuse: there was weak evidence that more severe hallucinations were associated with risk of disengagement among people without comorbid substance misuse (hazard ratio $1.64,95 \%$ CI 1.12-2.40) but not in those with a history of substance misuse (hazard ratio $1.06,95 \%$ CI $0.79-1.43$, LRT $P=0.069$ ).

In sensitivity analyses we re-ran our models, excluding anyone discharged early for reasons other than disengagement. This provided similar results (data available from authors), although the risk of disengagement became stronger with male gender (hazard ratio $1.70,95 \% \mathrm{CI} 1.03-2.80$ ) and hallucinations (hazard ratio 1.28 , 95\% CI 1.00-1.64). Results were similar when using logistic regression models, ignoring time in treatment (data available from authors).
Discussion

\section{Main findings}

This is the first epidemiological study in the UK to investigate sociodemographic and clinical predictors of disengagement from EIP services using a large naturalistic cohort sample. We found that over half of all clients accepted into EIP services in the East of England (54.3\%) did not complete 3 years of care, which may have considerable implications for achieving sustained long-term positive outcomes following early intervention. Nonetheless, the total number of clients discharged due to disengagement was lower (11.7\%). Disengagement was associated with being employed (or a student), not meeting diagnostic criteria for FEP during EIP care, having a history of polysubstance misuse or dependency, having lower levels of psychomotor poverty and fewer first-rank delusions.

\section{Meaning of the findings and comparisons with previous studies}

Previous studies have found a proportion of service disengagement ranging between $13^{8}$ and $31 \% .^{9}$ Although our study broadly aligns with these figures, differences in the definition of disengagement used across studies make direct comparisons difficult. ${ }^{22,23}$ For instance, we did not include patients who moved out of the catchment area $(n=34,4.33 \%)$ in our measure of disengagement; whereas one previous study did, ${ }^{10}$ reporting a disengagement level of $24 \%$ within 12 months of admission. If we used the same definition, albeit over a 3 -year period, we would have seen a total of $16 \%$ of patients disengaging. This suggests that disengagement from EIP services in the East of England may have been lower than previously found.

Nonetheless, Doyle et $a l^{22}$ and Lai and Malla ${ }^{23}$ have suggested that the heterogeneity in reported disengagement rates may partly result from the absence of an internationally recognised standard to define disengagement from EIP care. We note that other studies have used broad definitions of disengagement: discontinuation of EIP care despite on-going need ${ }^{8}$ (13\% patients disengaged), failure to present to services despite contact being made by case manager $^{11}$ (23\% patients disengaged), not making contact with EIP for three consecutive months (28\% disengagement $)^{24}$ and leaving the programme prior to the end of the 30 months of treatment ( $31 \%$ patients disengaged). ${ }^{9}$ The latter definition appears to be the broadest and it aligns closely with the rate of disengagement in our study when defined in this way (34\%), having excluded those who were discharged due to recovery. It is also possible that heterogeneity in these estimates reflects differences in healthcare systems in different countries; with the exception of a single trial, ${ }^{25}$ no study has been conducted in the UK. The lack of consistency in definition of disengagement from EIP services may hinder effective clinical service provision and makes comparability across research studies difficult. We suggest that policy guidance should be developed to allow clinicians and services to more accurately monitor and minimise service disengagement.

Despite differences in disengagement rates, our findings in relation to predictors of severity are largely in agreement with the previous literature, including lower symptom severity associated with greater disengagement. ${ }^{8-11,26}$ However, in contrast to earlier studies (which mainly used scales of overall ${ }^{8,11,26}$ or positive and negative ${ }^{9,10}$ symptoms), we found strong evidence of disengagement in participants with lower psychomotor poverty (i.e. negative symptoms) and fewer first-rank delusions (i.e. a positive symptom). Although this encouraging finding suggests that participants with more severe psychopathologies are successfully retained in EIP, it 
Table 1 Sample characteristics by disengagement from EIP care

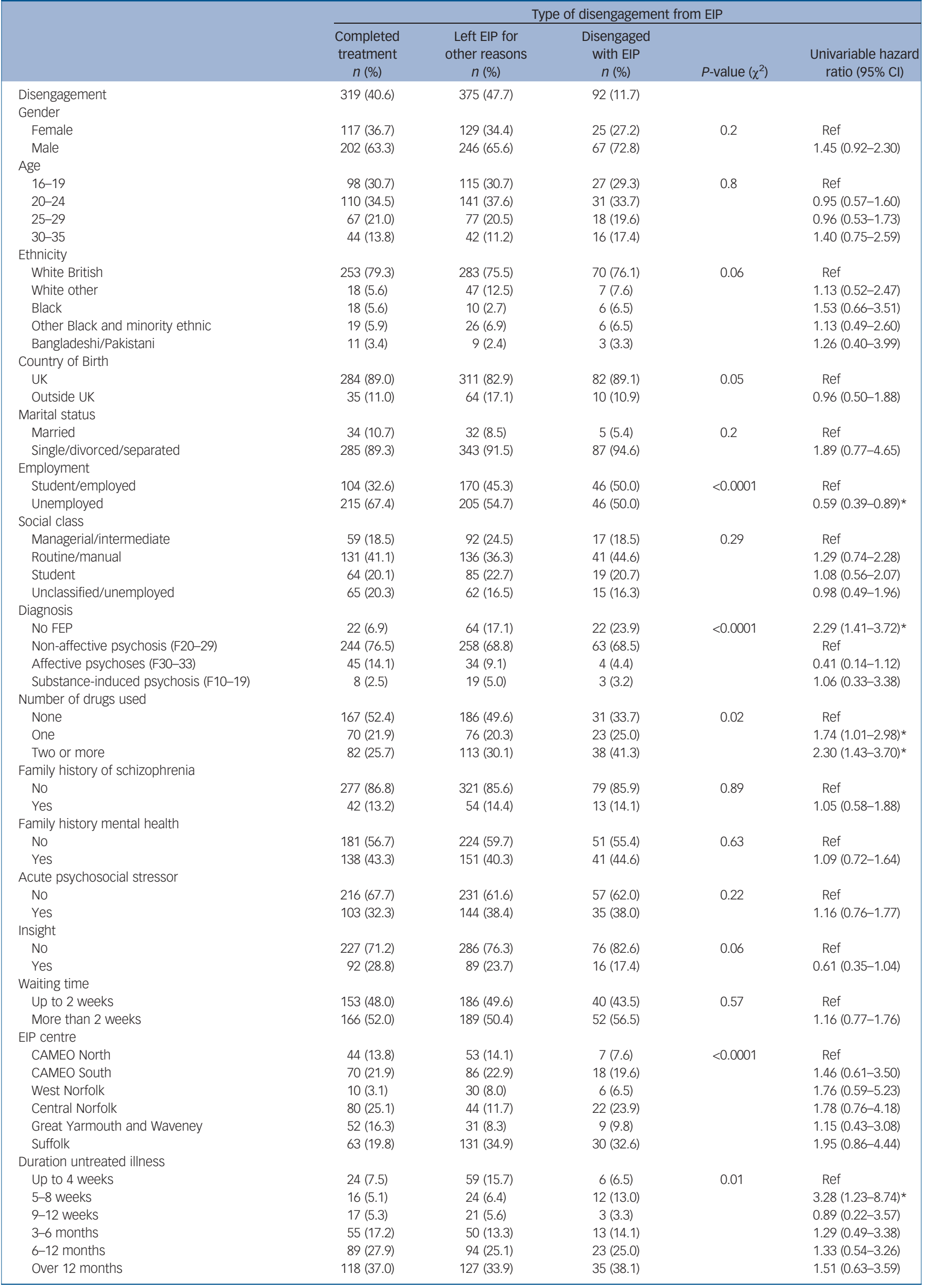

(Continued) 


\begin{tabular}{|c|c|c|c|c|c|}
\hline & Mean (s.d.) & Mean (s.d.) & Mean (s.d.) & $P$-value & $\begin{array}{l}\text { Univariable hazard } \\
\quad \text { ratio }(95 \% \mathrm{Cl})\end{array}$ \\
\hline Manic symptoms & $0.09(0.9)$ & $-0.05(1.1)$ & $-0.08(0.8)$ & 0.11 & $0.86(0.69-1.06)^{a}$ \\
\hline Depressive symptoms & $0.04(1.0)$ & $-0.03(0.9)$ & $-0.05(1.0)$ & 0.62 & $0.93(0.76-1.14)^{a}$ \\
\hline Delusions & $0.10(0.9)$ & $-0.09(0.9)$ & $-0.006(1.1)$ & 0.03 & $0.96(0.78-1.17)^{a}$ \\
\hline Hallucinations & $-0.02(1.0)$ & $-0.06(1.1)$ & $0.32(0.9)$ & 0.004 & $1.35(1.10-1.67)^{\mathrm{a} *}$ \\
\hline Paranoia & $0.03(1.1)$ & $-0.03(0.9)$ & $0.03(0.9)$ & 0.05 & $1.00(0.82-1.22)^{a}$ \\
\hline Psychomotor poverty & $0.05(1.1)$ & $0.01(0.9)$ & $-0.22(0.9)$ & 0.06 & $0.79(0.65-0.95)^{a *}$ \\
\hline First-rank delusions & $0.09(1.0)$ & $-0.01(1.0)$ & $-0.37(0.8)$ & 0.0005 & $0.71(0.59-0.86)^{a *}$ \\
\hline
\end{tabular}

nonetheless highlights that a proportion of people with milder initial presentations, or those who do not later meet diagnostic criteria for FEP, may be accepted onto EIP caseloads. This raises the possibility that those who present with less severe psychopathologies may be more likely to disengage early, as evidenced in our study among those in employment at baseline. These results are also consistent with the possibility that early intervention care resulted in symptom remission in some individuals, facilitating later disengagement. Although these participants may also have been false-positive presentations to EIP services, who may require signposting to other specialist mental health services, it is also possible that they represent a group of high-risk individuals for whom EIP care has been successful during the period of observation.

Research has consistently found greater disengagement rates among individuals with a history of substance misuse ${ }^{10}$ or who continue to use drugs while in treatment. ${ }^{11}$ This mirrors our observations: we found that patients with substance misuse or dependency (including alcohol) issues were more likely to disengage with services, with some evidence of a dose-response association between number of substances used and risk of disengagement. We also found that participants reporting more severe hallucinations were more likely to disengage, with this association being stronger in sensitivity analyses. Here, post hoc analyses provided some weak evidence that this effect was more pronounced in people without substance use than in those with comorbid substance misuse. Although this finding warrants further attention, it tentatively suggests that individuals with comorbid mental health or behavioural issues have additional needs which may result in less disengagement with EIP services. This may be because EIP services have more intensive or regular contact with participants that have more complex morbidities. Unfortunately, we did not have information on the extent (i.e. frequency and quality) of

\begin{tabular}{|c|c|c|}
\hline & $n(\%)$ & $\begin{array}{l}\text { Median duration } \\
\text { of treatment }\end{array}$ \\
\hline Completed 3 years of EIP care & $319(40.6 \%)$ & $35.9(35.1-36.0)$ \\
\hline Disengagement (primary outcome) & $92(11.7 \%)$ & $15.0(8.2-21.2)$ \\
\hline Moved out of area & $34(4.3 \%)$ & $5.6(3.8-10.4)$ \\
\hline No FEP & 27 (3.4\%) & $12.3(8.5-26.3)$ \\
\hline Recovery & $150(19.1 \%)$ & $15.8(10.3-25.6)$ \\
\hline Transferred to GP & $14(1.8 \%)$ & $20.0(14.7-20.7)$ \\
\hline Transferred to another EIP & $40(5.1 \%)$ & $8.9(4.0-18.1)$ \\
\hline Transfer to another mental health service & $56(7.1 \%)$ & $16.5(8.9-23.4)$ \\
\hline Other $^{a}$ & $54(6.9 \%)$ & $22.7(14.1-28.3)$ \\
\hline
\end{tabular}

engagement prior to discharge/disengagement, but our findings suggest this is an important area for future research. Nonetheless, over half of our sample had no documented history of substance misuse and those with more hallucinations had a higher risk of disengagement, underlining the need for EIP services to meet the heterogeneous needs of clients to prevent premature disengagement.

Finally, the high overall discharge rate (54.3\%) (vis-à-vis disengagement) highlights that only a minority of people accepted on caseloads complete the full EIP care package. Although several studies have shown that positive effects of EIP are often not sustained after patient discharge, ${ }^{13,15,27}$ a recent trial has found some evidence that individuals assigned to extended EIP - lasting 1 year longer than the usual care package - may have better longrun outcomes in terms of social functioning (e.g. full-time employment rate). ${ }^{15}$ This suggests that longer engagement with EIP care could result in greater long-term sustainability of its positive effects and to the need for greater effort to ensure patient retention in EIP care programmes. However, we also acknowledge that length of engagement is only one of several indices that are likely to determine clinical and social outcomes following EIP care: quality of engagement is also highly relevant and it is important that engagement also results in a meaningful therapeutic alliance. As Lai and Malla $^{23}$ have suggested, meaningful engagement is only likely to occur routinely when services can align their care offering to not only be efficacious but also 'caring, respectful, and nonjudgmental'. Further, they suggest clinicians 'need to be flexible in how, when, and where services are delivered, and which components of services are delivered over time.' We echo their call that disengagement should also be regarded as a multidimensional construct and that people may disengage from services in a variety of ways, or may only engage with certain aspects of care. All such issues will affect the quality of engagement and instruments that can assess such issues need to be developed, validated and deployed in future studies. $^{28,29}$

\section{Methodological considerations}

This study had a number of strengths. We employed data from a large naturalistic cohort of EIP clients across six sites, representing 98.5\% of all those people who met the inclusion criteria for the SEPEA study. At initial presentation and acceptance to services we included people who were later found not to meet a diagnosis of psychotic disorder, allowing us to identify this as a reason for disengagement. This has potential implications for service provision, as it suggests that better identification of 'at-risk' cases might result in more targeted and, potentially, cost-effective interventions. We included data on a large number of predictors, including clinical variables derived from OPCRIT, and raters were trained, showing good interrater reliability. ${ }^{18}$ However, several clinical predictors were derived from single OPCRIT items, which may have resulted in some degree of measurement error. Furthermore, although 
Table 3 Multivariable Cox proportional hazard model

Multivariable hazard ratio $(95 \% \mathrm{Cl})^{a}$

\section{Gender \\ Female \\ Male}

Ref

Marital Status

Married

Single, divorced or separated

Age

$16-19$

20-24

25-29

30-35

EIP centre

CAMEO North

CAMEO South

West Norfolk

Central Norfolk

Great Yarmouth and Waveney

Suffolk

Duration of untreated illness

Up to 4 weeks

5-8 weeks

9-12 weeks

3-6 months

6-12 months

Over 12 months

Employment

Student/employed

Unemployed

Diagnosis

No FEP

Non-affective psychosis (F20-29)

Affective psychoses (F30-33)

Substance-induced psychosis (F10-19)

Number of drugs used

None

One

Two or more

First-rank delusions ${ }^{b}$

Hallucinations

$1.17(0.68-2.01)$

$1.28(0.67-2.45)$

$1.88(0.95-3.70)^{*}$

Ref

$1.94(0.79-4.78)$

$1.70(0.55-5.20)$

$1.05(0.42-2.64)$

$0.88(0.30-2.53)$

$1.31(0.55-3.13)$

Ref

$5.19(1.85-14.56)^{* *}$

$0.78(0.19-3.210)$

$1.56(0.58-4.19)$

$1.51(0.59-3.86)$

$1.64(0.67-4.02)$

Ref

$0.44(0.28-0.69)^{* *}$

$2.52(1.49-4.26)^{* *}$

Ref

$0.39(0.14-1.13)^{*}$

$0.66(0.19-2.22)$

Ref

$1.66(0.94-2.94)^{*}$

$2.20(1.30-3.72)^{\star * *}$

$0.71(0.57-0.88)^{* *}$

$0.74(0.60-0.83)^{* *}$

$1.24(0.97-1.58)^{*}$
Psychomotor poverty ${ }^{b}$

discharged early, exhaustive methods were implemented to retain clients where possible and we carefully checked all cases for inconsistencies in coding, finding only one instance of misclassification.

Francesca Solmi, Research Associate, Division of Psychiatry, University College

London, UK; Abdolali Mohammadi, Specialty Doctor in Psychiatry, St. Ann's Hospital, London, UK; Jesus A. Perez, Consultant Psychiatrist, Department of Psychiatry,

University of Cambridge, UK, Cambridgeshire and Peterborough Foundation Trust and National Institute for Health Research Collaboration for Leadership in Applied Health Research and Care East of England, UK; Yasir Hameed, Honorary Lecturer, Norfolk and Suffolk Foundation Trust, UK: Peter B. Jones, Professor of Psychiatry, Department of Psychiaty, University of Cambridge, UK, Cambridgeshire and Peterbough Four

gh Foundation Trust and National Institute for Health Research Collaboration for Leadership in Applied Health Research and Care East of England, UK; James B. Kirkbride, Reader in Epidemiology, Division of Psychiatry, University College London, UK.

Ref, reference; EIP, Early Intervention in Psychosis; FEP, first episode of psychosis.

${ }^{\mathrm{b}}$ Hazard ratio above one indicates greater likelihood of being discharged.
${ }^{2}$ Change in risk (hazard) of being discharged due to disengagement associated with

${ }^{b}$ Change in risk (hazard) of
1 s.d. change in symptoms

${ }^{*} 0.1>P>0.05, * * P \leq 0.05$

extensive, we acknowledge that the list of predictors we could investigate did not include some factors that have previously been associated with disengagement from EIP services. For instance, although not unequivocally, ${ }^{24}$ evidence suggests that individuals who do not have a family member involved with treatment ${ }^{9}$ or who are not living with family ${ }^{11,26}$ disengage with EIP more frequently. In our study, we found weak evidence of greater disengagement among unmarried participants in univariable models, but we did not have data on family involvement. A recent review also highlighted that different pathways to care in individuals who experience FEP could be associated with greater stigma, poorer help-seeking attitudes and adherence to treatment. ${ }^{30}$ In our study we did not have information on pathways to care, although one previous study did not find evidence that this affected disengagement. ${ }^{24}$ We also did not have information on levels of social functioning and had limited power to explore associations with disengagement for some of our predictors (e.g., marital status and DUI), which could be proxies of functioning and is reflected in the imprecise estimates reported here. Our definition of early discharge due to disengagement was based on clinical information, which might have contained a degree of error. Nonetheless, for all participants
Correspondence Dr Francesca Solmi, PsyLife group, Division of Psychiatry, University College London, 6th Floor, Wing B, Maple House, 149 Tottenham Court Road, N1T 7NF, London, UK. Email: francesca.solmi@ucl.ac.uk

First received 12 Jan 2018, final revision 11 Apr 2018, accepted 12 Apr 2018

\section{Supplementary material}

Supplementary material is available online at https://doi.org/10.1192/bjp.2018.91.

\section{Funding}

The work for this paper was supported by a Sir Henry Wellcome Research Fellowship from the Wellcome Trust (grant no. WT085540) to J.B.K.; a Sir Henry Dale Fellowship to J.B.K., jointly funded by the Wellcome Trust and the Royal Society (grant no. 101272/Z/13/Z); and a National Institute for Health Research Collaboration for Leadership in Applied Health Research and care grant for Cambridgeshire and Peterborough (grant no. RP-PG-0606-1335) to P.B.J. The funders had no involvement in any aspect of the design of this study, preparation of results or decision to submit for publication.

\section{Acknowledgements}

We thank the Cambridgeshire and Peterborough Foundation Trust (CPFT) and Norfolk and Suffolk Foundation Trust (NSFT) for sponsoring this research. We are indebted to all patients and staff at the six Early Intervention in Psychosis services where the Social Epidemiology of Psychosis in East Anglia (SEPEA) study took place: CAMEO North (Peterborough, CPFT), CAMEO South (Cambridge, CPFT), the West Norfolk Early Intervention Service (Kings Lynn, NSET), the Central Norfolk Early Intervention Team (Nonwich, NSFT), the Great Yarmouth and Intervention in Psychosis Service (Stowmarket, NSFT) We thank the National Institute for Health Research Clinical Research Network: Eastern (formerly the Mental Health Research Network) for the invaluable support provided to the study. We are grateful for the dedicated help of all assistant psychologists and Clinical Studies Officers who contributed to data collection. We thank all clinicians who completed Operational Criteria Checklist for Psychotic Illness and Affective Illness (OPCRIT) assessments for the SEPEA study.

\section{References}

1 National Institute for Health and Care Excellence (NICE). Psychosis and Schizophrenia in Adults: Prevention and Management. NICE, 2014. Available at: http://nice.org.uk/guidance/cg178.

2 National Institute for Health and Care Excellence (NICE). Implementing the Early Intervention in Psychosis Access and Waiting Time Standard: Guidance. NHS England, 2016.

3 Marshall M, Lewis S, Lockwood A, Drake R, Jones P, Croudace T. Association between duration of untreated psychosis and outcome in cohorts of firstepisode patients. Arch Gen Psychiatry 2005; 62: 975-83.

4 McGorry PD. Early intervention in psychosis. J Nerv Ment Dis 2015; 203: 310-8.

5 Stafford MR, Jackson H, Mayo-Wilson E, Morrison AP, Kendall T. Early interventions to prevent psychosis: systematic review and meta-analysis. $B M J$ 2013; 346: f762.

6 Mihalopoulos C, Harris M, Henry L, Harrigan S, McGorry P. Is early intervention in psychosis cost-effective over the long term? Schizophr Bull 2009; 35 909-18.

7 Mccrone P, Craig TKJ, Power P, Garety PA. Cost-effectiveness of an early 377-82.

8 Chan TCW, Chang WC, Hui CLM, Chan SKW, Lee EHM, Chen EYH. Rate and predictors of disengagement from a 2-year early intervention program for psychosis in Hong Kong. Schizophr Res 2014; 153: 204-8. intervention service for people with psychosis. Br J Psychiatry 2010; 196: 
9 Stowkowy J, Addington D, Liu L, Hollowell B, Addington J. Predictors of disengagement from treatment in an early psychosis program. Schizophr Res 2012; 136: 7-12.

10 Turner M, Smith-Hamel C, Mulder R. Prediction of twelve-month service disengagement from an early intervention in psychosis service. Early Interv Psychiatry 2007; 1: 276-81.

11 Conus P, Lambert M, Cotton S, Bonsack C, McGorry PD, Schimmelmann BG. Rate and predictors of service disengagement in an epidemiological firstepisode psychosis cohort. Schizophr Res 2010; 118: 256-63.

12 Albert N, Melau M, Jensen $\mathrm{H}$, Emborg C, Jepsen JRM, Fagerlund B, et al. Five years of specialised early intervention versus two years of specialised early intervention followed by three years of standard treatment for patients with a first episode psychosis: randomised, superiority, parallel group trial in Denmark (OPUS II). BMJ 2017; 356: j1015

13 Bertelsen $\mathrm{M}$, Jeppesen $\mathrm{P}$, Petersen $\mathrm{L}$, Thorup $\mathrm{A}$, Øhlenschlæger J, le Quach $\mathrm{P}$ et al. Five-year follow-up of a randomized multicenter trial of intensive early intervention vs standard treatment for patients with a first episode of psychotic illness. Arch Gen Psychiatry 2008; 65: 762.

14 Chang WC, Chan GHK, Jim OTT, Lau ESK, Hui CLM, Chan SKW, et al. Optimal duration of an early intervention programme for first-episode psychosis: randomised controlled trial. Br J Psychiatry 2015; 206: 492-500.

15 Chang WC, Kwong VWY, Lau ESK, So HC, Wong CSM, Chan GHK, et al. Sustainability of treatment effect of a 3-year early intervention programme for first-episode psychosis. Br J Psychiatry 2017; 211: 37-44.

16 Leclerc E, Noto C, Bressan RA, Brietzke E. Determinants of adherence to treatment in first-episode psychosis: a comprehensive review. Rev Bras Psiquiatr 2015; 37: 168-76.

17 Kreyenbuhl J, Nossel IR, Dixon LB. Disengagement from mental health treatment among individuals with schizophrenia and strategies for facilitating connections to care: a review of the literature. Schizophr Bull 2009; 35: 696-703.

18 Kirkbride JB, Hameed Y, Ankireddypalli G, Ioannidis K, Crane CM, Nasir M, et al. The epidemiology of first-episode psychosis in early intervention in psychosis services: findings from the social epidemiology of psychoses in East Anglia [SEPEA] study. Am J Psychiatry 2016; 174: 143-53.
19 McGuffin P, Farmer A, Harvey I. A polydiagnostic application of operational criteria in studies of psychotic illness: development and reliability of the OPCRIT system. Arch Gen Psychiatry 1991; 48: 764-70.

20 Williams J, Farmer AE, Ackenheil M, Kaufmann CA, McGuffin P. A multicentre inter-rater reliability study using the OPCRIT computerized diagnostic system. Psychol Med 1996; 26: 775-83.

21 StataCorp. Stata Statistical Software version 13. 2013

22 Doyle R, Turner N, Fanning F, Brennan D, Renwick L, Lawlor E, et al. Firstepisode psychosis and disengagement from treatment: a systematic review. Psychiatr Serv 2014; 65: 603-11.

23 Lal S, Malla A. Service engagement in first-episode psychosis: current issues and future directions. Can. J. Psychiatry 2015; 60: 341-5.

24 Anderson KK, Fuhrer R, Schmitz N, Malla AK. Determinants of negative pathways to care and their impact on service disengagement in first-episode psychosis. Soc Psychiatry Psychiatr Epidemiol 2013; 48: 125-36.

25 Craig TKJ, Garety P, Power P, Rahaman N, Colbert S, Fornells-Ambrojo M, et al. The Lambeth Early Onset (LEO) Team: randomised controlled trial of the effectiveness of specialised care for early psychosis. BMJ 2004; 329: 1067

26 Schimmelmann BG, Conus $\mathrm{P}$, Schacht M, Mcgorry $\mathrm{P}$, Lambert M. Predictors of service disengagement in first-admitted adolescents with psychosis. J Am Acad Child Adolesc Psychiatry 2006; 45: 990-9.

27 Gafoor R, Nitsch D, McCrone P, Craig TKJ, Garety PA, Power P, et al. Effect of early intervention on 5-year outcome in non-affective psychosis. Br J Psychiatry 2010; 196: 372-6.

28 Tait L, Birchwood M, Trower P. A new scale (SES) to measure engagement with community mental health services. J Ment Heal 2002; 11: 191-8.

29 Tetley A, Jinks M, Huband N, Howells K. A systematic review of measures of therapeutic engagement in psychosocial and psychological treatment. J Clin Psychol 2011; 67: 927-41.

30 Gronholm PC, Thornicroft G, Laurens KR, Evans-Lacko S. Mental health-related stigma and pathways to care for people at risk of psychotic disorders or experiencing first-episode psychosis: a systematic review. Psychol Med 2017; 47: $1867-79$

\section{No account of post-traumatic recurrent dreams in the second century AD}

\section{Greg Wilkinson}

Artemidorus, ${ }^{1}$ whose books encompass a system of dream interpretation in the second century AD, was overwhelmed by the vast amount of research involved in the work and by the abundance of material. He adds that not only has he taken special pains to procure every book on the interpretation of dreams, but he has consulted for many years with the diviners of the marketplace. Although trauma is likely to have been highly prevalent, Artemidorus gives no account of post-traumatic dreams. This suggests that these dreams were not a feature of his wide reading, consultation and practice, but rather that they are a product of later cultures:

'Recurrent dreams, if they appear at small intervals and continually, should be considered as always having the same meaning. And because they are seen frequently, we should be more attentive to them and place greater faith in them. As a matter of fact, whenever we have something important to say, we usually say it frequently. Similarly, then, our mind also presents these dreams frequently either because it is prophesying matters that are serious, meaningful and not of secondary importance or because it has begun to see them long before their fulfilment and continues to see them uninterruptedly.

But whenever the intervals between recurrent dreams are long, one must realise that the dreams will mean different things at different times. For just as the same dream comes true in different ways for each person who has seen it because the circumstances of all men are not the same, so too the same dream when seen at different times by one person will come true in different ways because he himself does not always remain in the same circumstances.

For example, a man dreamt that he lost his nose. He was a perfume dealer at the time. Since he did not have a nose in the dream, he lost his store and stopped selling perfumes. For he no longer possessed the means to test his perfumes and it was obvious that he would not continue in the perfume business. When he was no longer a perfume dealer, the same man dreamt thathe did not have a nose. He was caught forging a signature and fled his own country. For anything that is lacking to a face disfigures and degrades it. And the face is the image of one's respectability and reputation. It is understandable that this man was disgraced.

During an illness the same man dreamt that he did not have a nose. He died not long afterwards, for the skull of the dead man has no nose. The first time, when he was a merchant the dream referred to his perfumes. The second time, when he was a citizen with full rights and franchises it referred to his reputation. The third time, when he was sick, it referred to his body itself. In this way, then, the same dream came true in three different ways for the same man’.

\section{Reference}

1 Artemidorus Daldianus. Oneirocritica. [The interpretation of dreams.] (translation and commentary by Robert J. White): 197-8. Noyes Press, 1975. 